\title{
Protective effects of endothelial progenitor cell microvesicles on Ang II-induced rat kidney cell injury
}

\author{
YANLING SONG $^{1 *}$, ZHENBING BAI $^{2 *}$, YUANYUAN ZHANG $^{3}$, JUMING CHEN $^{1}$, MINGHUI CHEN $^{1}$, \\ YUNBO ZHANG ${ }^{1}$, XIAODIAN ZHANG ${ }^{4-6}$, HUADE MAI $^{1}$, BINGSHU WANG $^{4-6}$, \\ YUNYUN LIN $^{1}$ and SHENHONG GU ${ }^{1}$ \\ ${ }^{1}$ Department of General Practice, The First Affiliated Hospital of Hainan Medical University; ${ }^{2}$ Hainan Medical University; \\ ${ }^{3}$ Department of Cardiology; ${ }^{4}$ Key Laboratory of Tropical Cardiovascular Diseases Research of Hainan Province, \\ The First Affiliated Hospital of Hainan Medical University; ${ }^{5}$ Key Laboratory of Emergency and Trauma of \\ Ministry of Education, Hainan Medical University; ${ }^{6}$ Research Unit of Island Emergency Medicine of \\ Chinese Academy of Medical Sciences, Hainan Medical University, Haikou, Hainan 570100, P.R. China
}

Received March 31, 2021; Accepted June 14, 2021

DOI: $10.3892 / \mathrm{mmr} .2021 .12520$

\begin{abstract}
Chronic hypertension can lead to kidney damage, known as hypertensive nephropathy or hypertensive nephrosclerosis. Further understanding of the molecular mechanisms via which hypertensive nephropathy develops is essential for effective diagnosis and treatment. The present study investigated the mechanisms by which endothelial progenitor cells (EPCs) repair primary rat kidney cells (PRKs). ELISA, Cell Counting Kit-8 and flow cytometry assays were used to analyze the effects of EPCs or EPC-MVs on the oxidative stress, inflammation, cell proliferation, apoptosis and cycle of PRKs induced by AngII. A PRK injury model was established using angiotensin II (Ang II). After Ang II induction, PRK proliferation was decreased, apoptosis was increased and the cell cycle was blocked at the $\mathrm{G}_{1}$ phase before entering the $\mathrm{S}$ phase. It was found that the levels of reactive oxygen species and malondialdehyde were increased, while the levels of glutathione peroxidase and superoxide dismutase were decreased. Moreover, the levels of the inflammatory cytokines
\end{abstract}

Correspondence to: Dr Shenhong Gu, Department of General Practice, The First Affiliated Hospital of Hainan Medical University, Outpatient and Emergency Building, 31 Longhua Road, Haikou, Hainan 570100, P.R. China

E-mail: renee388@163.com

*Contributed equally

Abbreviations: Ang II, Angiotensin II; ROS, reactive oxygen species; EPCs, endothelial progenitor cells; PRKs, primary rat kidney cells; MVs, microvesicles; MDA, malondialdehyde; GSH-Px, glutathione peroxidase; SOD, superoxide dismutase; FCM, flow cytometry; CCK-8, Cell Counting Kit-8; Dil-Ac-LDL, Dil complex acetylated low-density lipoprotein

Key words: EPC-MVs, hypertensive nephropathy, Ang II, ROS, MVs, inflammation
IL-1 $\beta$, IL-6 and TNF- $\alpha$ were significantly increased. Thus, Ang II damaged PRKs by stimulating oxidative stress and promoting the inflammatory response. However, when PRKs were co-cultured with EPCs, the damage induced by Ang II was significantly reduced. The current study collected the microvesicles (MVs) secreted by EPCs and co-cultured them with Ang II-induced PRKs, and identified that EPC-MVs retained their protective effect on PRKs. In conclusion, EPCs protect PRKs from Ang II-induced damage via secreted MVs.

\section{Introduction}

Hypertension is a risk factor for the incidence of cardiovascular and cerebrovascular diseases, and their associated mortality (1). The kidneys can cause hypertension and are one of the target organs of hypertension damage (2). Chronic kidney disease (CKD) has been one of the major causes of increased mortality among individuals with high blood pressure worldwide over the past two decades $(3,4)$. Thus, there is an urgent necessity to promote the treatment of hypertensive nephropathy and study its pathological mechanism.

Previous studies have shown that angiotensin II (Ang II) induced vascular damage and served a key role in vascular diseases via several mechanisms, such as inducing apoptosis (5), cell cycle arrest (6), increasing reactive oxygen species (ROS) levels (7), promoting the oxidative stress response by increasing the secretion of malondialdehyde (MDA), and reducing the secretion of glutathione peroxidase (GSH-Px) and superoxide dismutase (SOD) $(8,9)$. Ang II also promotes the production of inflammation-related factors, such as IL-6, IL-1 $\beta$ and TNF- $\alpha$ (10-12). Ang II-induced damage has therefore been used to model hypertension in vitro, including in primary rat kidney cells (PRKs) $(13,14)$.

For patients with renal function impairment, damage to endothelial cells and decreased regeneration and repair are the main causes of the loss of peritubular microvesicles (MVs) (15). Endothelial progenitor cells (EPCs) derived from bone marrow can promote endothelial repair $(16,17)$. Previous 
studies have revealed that EPCs can improve cardiovascular regeneration (18) and regulate angiogenesis (19), as well as exert therapeutic effects on acute renal ischemia-reperfusion injury (20) and in patients with renal transplantation (21). However, to the best of our knowledge, the effect of EPCs on hypertensive nephropathy has not been previously reported. We hypothesized that EPCs may have a protective effect against hypertensive nephropathy in renal cells.

MVs are secreted continuously by a variety of cells in the body, such as epithelial cells, tumor cells and stem cells, and exist in several body fluids, such as blood, urine and milk, where they mediate biological functions (22). Accumulating evidence has suggested that the protective effect of EPCs is closely associated with the release of MVs $(23,24)$.

In the present study, the potential application of EPC-MVs for the treatment of hypertensive nephropathy was examined by investigating the protective effects and mechanisms via which EPC-MVs protect against Ang II-induced PRK injury, to provide a biological theoretical basis for the treatment of hypertensive nephropathy.

\section{Materials and methods}

Animals. A total of 12 8-week-old male Wistar-Kyoto (WKY) specific pathogen-free rats (weight, 80-120 g) were obtained from the Beijing Vital River Laboratory Animal Technology Co., Ltd. (license no. SCXK, Guangdong, 2015-0063). The rats were placed in a room with a constant temperature and humidity (temperature, $23 \pm 2^{\circ} \mathrm{C}$; humidity, $50 \pm 10 \%$ ), under a 12-h light/dark cycle with ad libitum access to standard rat food and water in a polystyrene cage. All animal experiments were approved by the Animal Care and Use Committee of Hainan Medical University (Haikou, China; approval no. HYLL-2021-053), and were performed according to the National Institutes of Health guidelines (25).

Isolation and culture of PRKs. WKY rats with free access to water were fasted for $12 \mathrm{~h}$ before the experiment. WKY mice were euthanized using an intraperitoneal injection of pentobarbital sodium ( $200 \mathrm{mg} / \mathrm{kg}$ body weight), then the kidneys were removed aseptically, and the cortical portion of the kidney was excised and transplanted into a small beaker. The renal cortex was cut into tissue fragments of $\sim 1 \mathrm{~mm}^{3}$, washed thrice with PBS (Gibco; Thermo Fisher Scientific, Inc.), centrifuged at $1,000 \mathrm{x}$ g at $25^{\circ} \mathrm{C}$ for $5 \mathrm{~min}$ and the supernatant was discarded. The tissue fragments were added to type I collagenase (Gibco; Thermo Fisher Scientific, Inc.) at a final concentration of $1 \mathrm{~g} / 1$, and the tissue fragments were oscillated and digested at $37^{\circ} \mathrm{C}$ for $30 \mathrm{~min}$. After filtration through a 200 -mesh $(0.075 \mathrm{~mm})$ stainless steel screen, the cells were separated by Ficoll (Sigma-Aldrich; Merck KGaA) density gradient centrifugation (26). After centrifugation at $1,000 \mathrm{x} \mathrm{g}$ at $25^{\circ} \mathrm{C}$ for $2 \mathrm{~min}$, the intermediate suspension was collected and mixed with MEM/F12 medium (Gibco; Thermo Fisher Scientific, Inc.), inoculated in a 6-well plate and cultured at $37^{\circ} \mathrm{C}$ under $5 \% \mathrm{CO}_{2}$. After $24 \mathrm{~h}$, the medium was removed and replaced with fresh medium, and the unattached renal cells and tissue were discarded. After $48 \mathrm{~h}$, the medium was removed again, the cells were washed twice with PBS and were denoted as PRKs $(27,28)$; the main constitute was rat glomerular mesangial cells. PRKs were cultured for three generations and treated with Ang II ( $1 \mu \mathrm{M}$; Sigma-Aldrich; Merck KGaA) at $37^{\circ} \mathrm{C}$ for $24 \mathrm{~h}$ to establish the PRK renal damage model. The Ang II-induced inflammation in rat renal tubular epithelial cells was performed as previously described by Nair et al (29).

PRKs were identified using an immunofluorescence (IF) assay. After rinsing thrice with PBS and fixing with 4\% paraformaldehyde at $25^{\circ} \mathrm{C}$ for $1 \mathrm{~h}$, the cells were incubated $\left(25^{\circ} \mathrm{C}\right)$ with Triton X-100 for $2 \mathrm{~h}$, followed by 5\% BSA (Beijing Solarbio Science \& Technology Co., Ltd.) at $25^{\circ} \mathrm{C}$ for $30 \mathrm{~min}$. The PRKs were then incubated overnight in darkness with $\alpha$-smooth muscle actin ( $\alpha$-SMA; $1 \mu \mathrm{g} / \mathrm{ml}$; cat. no. ab7817; Abcam) and vimentin (1:250; cat. no. ab92547; Abcam) at $4^{\circ} \mathrm{C}$. After rinsing thrice with PBS, the cells were incubated with Alexa Fluor ${ }^{\circledR}$ 488- (1:100; cat. no. ab150077; Abcam) or 647-labeled (1:200; cat. no. ab150075; Abcam) secondary antibodies at $37^{\circ} \mathrm{C}$ for $1 \mathrm{~h}$. Then, the cells were stained with DAPI $(0.5 \mu \mathrm{g} / \mathrm{ml}$; Beyotime Institute of Biotechnology) at $25^{\circ} \mathrm{C}$ for 5 min. Finally, images were captured using a fluorescence microscope (magnification, x400; Leica Microsystems GmbH).

Culture and identification of EPCs. The rat femur and tibia were dissected and each bone marrow tube was flushed with sterile PBS. The resulting mixture was centrifuged $(1,000 \mathrm{x} \mathrm{g}$; $25^{\circ} \mathrm{C} ; 15 \mathrm{~min}$ ) and the particles were re-suspended in MEM/F12 medium. Cells were isolated by Ficoll (Sigma-Aldrich; Merck $\mathrm{KGaA})$ density gradient centrifugation $\left(1,000 \mathrm{x} \mathrm{g} ; 25^{\circ} \mathrm{C}\right.$; $20 \mathrm{~min}$ ) and placed in a $25-\mathrm{cm}^{2}$ culture flask coated with fibronectin (BD Biosciences). Cells were maintained in a complete medium under standard conditions $\left(37^{\circ} \mathrm{C} ; 5 \% \mathrm{CO}_{2}\right)$. The culture medium was changed after 4 days, and the adherent cells were cultured for an additional 3 days (30). Dil complex acetylated low-density lipoprotein (Dil-Ac-LDL) staining (Beijing Solarbio Science \& Technology Co., Ltd.) was used to identify EPCs. For the co-culture system, PRKs $\left(1 \times 10^{4}\right)$ were added to the lower chamber of a co-cultivation plate (Corning, Inc.) for $24 \mathrm{~h}$ and then Ang II $(1 \mu \mathrm{M})$ or $200 \mu \mathrm{l} \mathrm{PBS}$ and EPCs $\left(3 \times 10^{4}\right)$ were added to the upper chamber and incubated at $37^{\circ} \mathrm{C}$ for $24 \mathrm{~h}$ before further cell function analysis was performed. When screening PRKs and the best proportion of EPCs, the cell number proportions were 2:1, 1:1, 1:2, 1:3, 1:4, 1:5, 1:6 and 1:7.

Preparation of EPC-MVs. EPCs were cultured for 7 days as previously described (30), washed twice with PBS and serum-starved for $12 \mathrm{~h}$. Subsequently, MEM/F12 medium containing cultured EPCs was collected and centrifuged at $4^{\circ} \mathrm{C}(1,000 \mathrm{x} \mathrm{g} ; 15 \mathrm{~min})$, and the supernatant was extracted at $4^{\circ} \mathrm{C}(100,000 \times \mathrm{g} ; 60 \mathrm{~min})$ for the collection of secreted EPC-MVs. After embedding in Pon 812 epoxy resin (Structure Probe, Inc.), it was placed at $37^{\circ} \mathrm{C}$ overnight. Then, electron microscope fixation solution (Wuhan Servicebio Technology Co., Ltd.) was added for $4 \mathrm{~h}$, and uranium acetate (2\%; Wuhan Servicebio Technology Co., Ltd.) and lead citrate (Wuhan Servicebio Technology Co., Ltd.) were stained at $25^{\circ} \mathrm{C}$ for $15 \mathrm{~min}$. MVs were confirmed via transmission electron microscopy. In the co-culture system, $50 \mu \mathrm{g} / \mathrm{ml}$ EPC-MVs (31) was added to the top chamber of a Transwell assay plate, and PRKs were added to the bottom chamber as previously described, and incubated for $24 \mathrm{~h}$. 
Cell proliferation assay. PRKs were digested with trypsin,

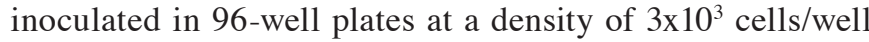
and cultured for $24 \mathrm{~h}$. The optical density at $450 \mathrm{~nm}$ was measured using $10 \mu \mathrm{l}$ Cell Counting Kit- 8 reagent (CCK-8; Beijing Solarbio Science \& Technology Co., Ltd.) at $37^{\circ} \mathrm{C}$ for $60 \mathrm{~min}$, as per the manufacturer's instructions, to evaluate the cell proliferation rate.

Cell apoptosis assay. A cell apoptosis assay was performed using an Annexin V-FITC Apoptosis Detection kit (BD Biosciences), according to the manufacturer's instructions. PRKs were harvested, washed twice with ice-cold PBS (Gibco; Thermo Fisher Scientific, Inc.) and resuspended in $500 \mu \mathrm{l}$ binding buffer. The resuspended PRKs were then incubated with $5 \mu \mathrm{l}$ Annexin V-FITC and $5 \mu \mathrm{l}$ PI in the dark for $15 \mathrm{~min}$ at $23 \pm 2^{\circ} \mathrm{C}$. Cell apoptosis was assessed using flow cytometry (FCM; FACSCanto II; BD FACSChorus ${ }^{\mathrm{TM}}$ software, version: 1.0; BD Biosciences).

EPC-MV and PRK fusion. To observe whether EPC-MVs fused with PRKs, EPC-MVs were labeled with a lipid membrane-embedded fluorescent dye (PKH26) prior to co-incubation. Briefly, $50 \mu \mathrm{g} / \mathrm{ml}$ EPC-MVs were combined with $2 \mathrm{ml} \mathrm{PKH26}\left(2 \times 10^{-6} \mathrm{M}\right.$; Sigma-Aldrich; Merck KGaA) and mixed at $23 \pm 2^{\circ} \mathrm{C}$ for $5 \mathrm{~min}$. The labeled mixture was added to $2 \mathrm{ml} 1 \% \mathrm{BSA}(\mathrm{v})$ and centrifuged at $100,000 \mathrm{x} \mathrm{g}$ at $4^{\circ} \mathrm{C}$ for $60 \mathrm{~min}$, then rinsed with cold PBS. The precipitate was suspended in $1 \mathrm{ml} \mathrm{MEM/F12} \mathrm{medium,} \mathrm{added} \mathrm{to} \mathrm{PRKs}$ and incubated at $37^{\circ} \mathrm{C}$ for $24 \mathrm{~h}$. Finally, $1 \mu \mathrm{g} / \mathrm{ml}$ DAPI was added for nuclear staining at $25^{\circ} \mathrm{C}$ for $15 \mathrm{~min}$. Cell images were acquired using a fluorescence microscope (magnification, x400; Leica Microsystems $\mathrm{GmbH}$ ).

Cell cycle assay. The cell cycle assay was performed using the Cell Cycle Detection kit (BD Biosciences). EPCs or PRKs $\left(1 \times 10^{6}\right)$ were harvested, washed twice with PBS and then fixed in $500 \mu 170 \%$ ice-cold ethanol for $2 \mathrm{~h}$ at $25^{\circ} \mathrm{C}$. The cells were then washed twice with cold PBS and incubated in PI $(400 \mu \mathrm{l})$ and RNase $(100 \mu \mathrm{l})$ for $30 \mathrm{~min}$ at $37^{\circ} \mathrm{C}$ in the dark. The PI signal was detected using FCM (FACSCanto II). The percentages of the cells in $\mathrm{G}_{1}, \mathrm{~S}$ and $\mathrm{G}_{2}$ phase were counted and compared using FlowJo software (version 10.0.6; FlowJo LLC).

ROS measurement by FCM. The PRKs were cultured to $60-70 \%$ confluency and harvested via trypsinization. All cells were incubated with $1.0 \mu \mathrm{M} 2^{\prime}, 7^{\prime}$-dichlorodihydrofluorescein diacetate for $15 \mathrm{~min}$ at $37^{\circ} \mathrm{C}$. Cells were then washed twice with PBS and analyzed via FCM to detect ROS using a $488 \mathrm{~nm}$ laser for excitation and a $535 \mathrm{~nm}$ laser for detection.

ELISA. After centrifugation $\left(1,000 \mathrm{x}\right.$; $\left.25^{\circ} \mathrm{C} ; 5 \mathrm{~min}\right)$, the cell supernatant of each subgroup was collected to analyze the levels of MDA, GSH-Px, SOD and the inflammatory factors IL-6, IL-1 $\beta$ and TNF- $\alpha$. The following kits were used according to the manufacturer's instructions: Micro MDA assay kit (cat. no. BC0020; Beijing Solarbio Science \& Technology Co., Ltd.), GSH-Px assay kit (cat. no. BC1195; Beijing Solarbio Science \& Technology Co., Ltd.), SOD activity detection kit (cat. no. BC0170; Beijing Solarbio Science \& Technology Co., Ltd.), rat IL-1 $\beta$ ELISA kit (cat. no. RLB00; R\&D Systems,
Inc.), rat IL-6 Quantikine ELISA kit (cat. no. R6000B; R\&D Systems, Inc.) and rat $\mathrm{TNF} \alpha$ Quantikine ELISA kit (cat. no. RTA00; R\&D Systems, Inc.).

Statistical analysis. All experiments were repeated thrice, and all data are expressed as the mean \pm SD. Data were analyzed using SPSS software version 21.0 (IBM Corp.). Differences between multiple groups were assessed using one-way ANOVA and Dunnett's post hoc test. $\mathrm{P}<0.05$ was considered to indicate a statistically significant difference.

\section{Results}

Identification of isolated EPCs and PRKs. The isolated EPCs were identified using Dil-Ac-LDL staining (Fig. 1A). Microscopic counting revealed that the degree of coincidence between red (Dil-Ac-LDL staining) and blue (nuclear staining with DAPI) fluorescence was $>90 \%$, confirming that EPCs were successfully isolated. Isolated PRKs were analyzed via IF (vimentin/ $\alpha-S M A)$. The results demonstrated that the expression level of vimentin/ $\alpha-S M A$ in PRKs was high and positive, suggesting the successful isolation of PRKs (Fig. 1B).

Optimal ratio of EPCs to PRKs. The effect of combining different proportions of PRKs/EPCs in a co-culture system were examined, and it was found that PRKs grown at a ratio of 1:3 PRKs/EPCs did not have increased proliferation rates compared with PRKs grown with a $2: 1,1: 1$ or $1: 2$ ratio of PRKs/EPCs (Fig. 1C). Moreover, increasing proportions of EPCs (1:4, 1:5, 1:6 and 1:7) resulted in a significant increase in PRK proliferation compared with that of EPCs (1:3). Therefore, a ratio of 1:3 PRKs/EPCs was selected for further studies to prevent possible interference from excessive EPCs in subsequent experiments.

Effect of EPC co-culture on Ang II-induced damage. PRKs $\left(1 \times 10^{4}\right)$ were inoculated $24 \mathrm{~h}$ in advance into the lower compartment of a Transwell plate. The upper chamber contained $200 \mu \mathrm{l}$ PBS in the control group and $200 \mu \mathrm{l}$ EPCs $\left(3 \times 10^{4}\right)$ in the experimental group. To model kidney damage, $1 \mu \mathrm{M}$ Ang II was added to the lower compartment. After $24 \mathrm{~h}, \mathrm{CCK}-8$ and FCM assays were performed to measure the proliferation rate of PRKs. PRKs receiving Ang II in the absence of EPCs had a significantly decreased $(\mathrm{P}<0.05)$ proliferation rate (Fig. 1D), and increased apoptosis rate (Fig. 1E) and cell cycle arrested in the $\mathrm{G}_{1}$ phase (Fig. 1F) compared with control group, while PRKs co-cultured with EPCs receiving Ang II showed the reverse effects. These results indicated that co-culture with EPCs can reduce the damage induced by Ang II in a rat kidney cell model.

To further understand the mechanisms of action by which EPCs protect PRKs from Ang II-induced damage, the levels of oxidative stress induced by Ang II in PRKs co-cultured with EPCs were examined. The results from the FCM assay revealed that Ang II significantly increased $(\mathrm{P}<0.05)$ the levels of ROS in PRKs in the absence of EPCs, while PRKs co-cultured with EPCs had comparatively lower ROS levels (Fig. 2A). Similarly, the ELISA results indicated that the levels of secreted MDA (Fig. 2B) were increased and GSH and SOD (Fig. 2C and D) levels were decreased in PRKs receiving Ang II, while PRKs receiving Ang II and co-cultured with EPCs showed 
A
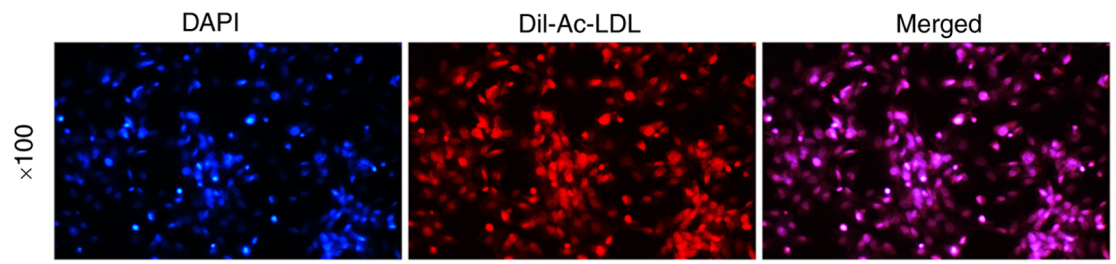

B
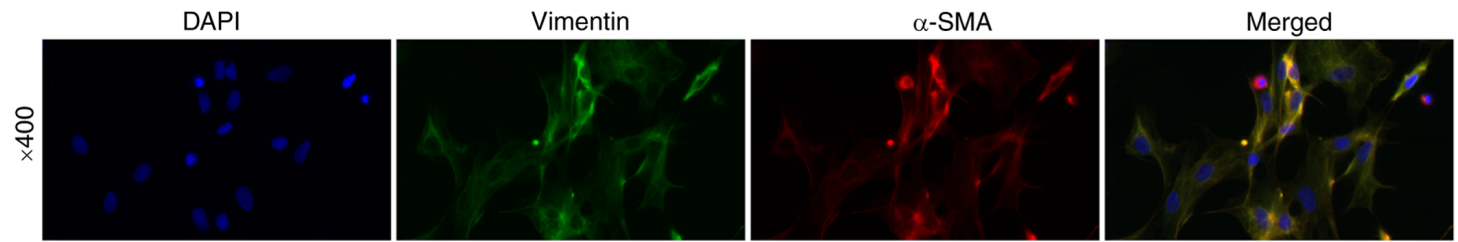

C

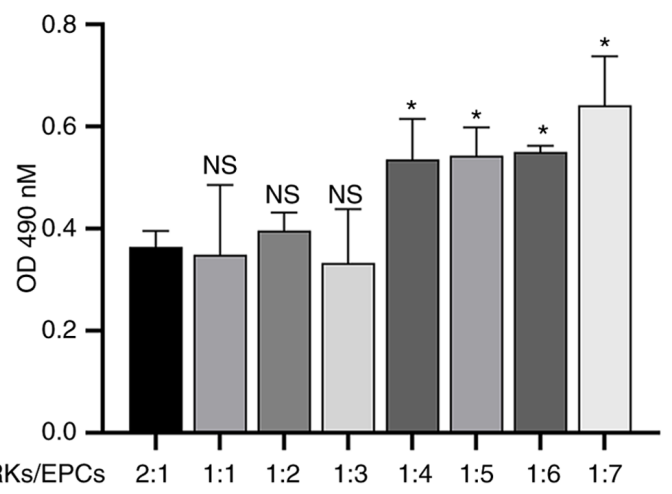

E

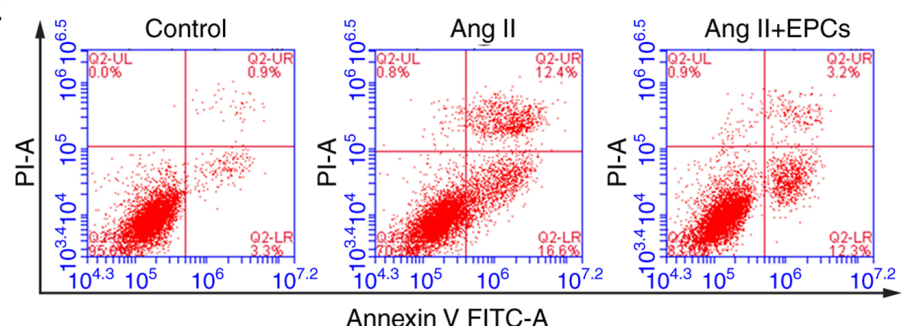

$\mathrm{F}$

Control

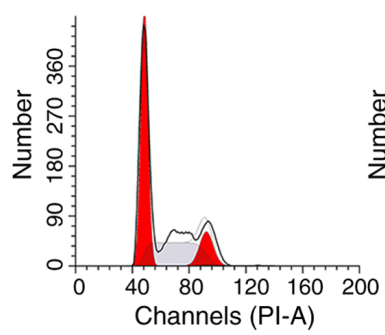

Ang II

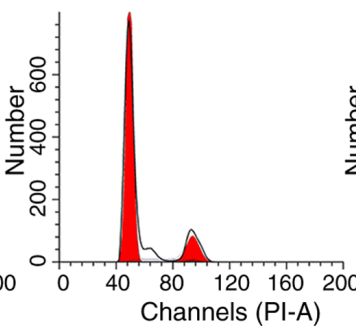

Ang II+EPCs
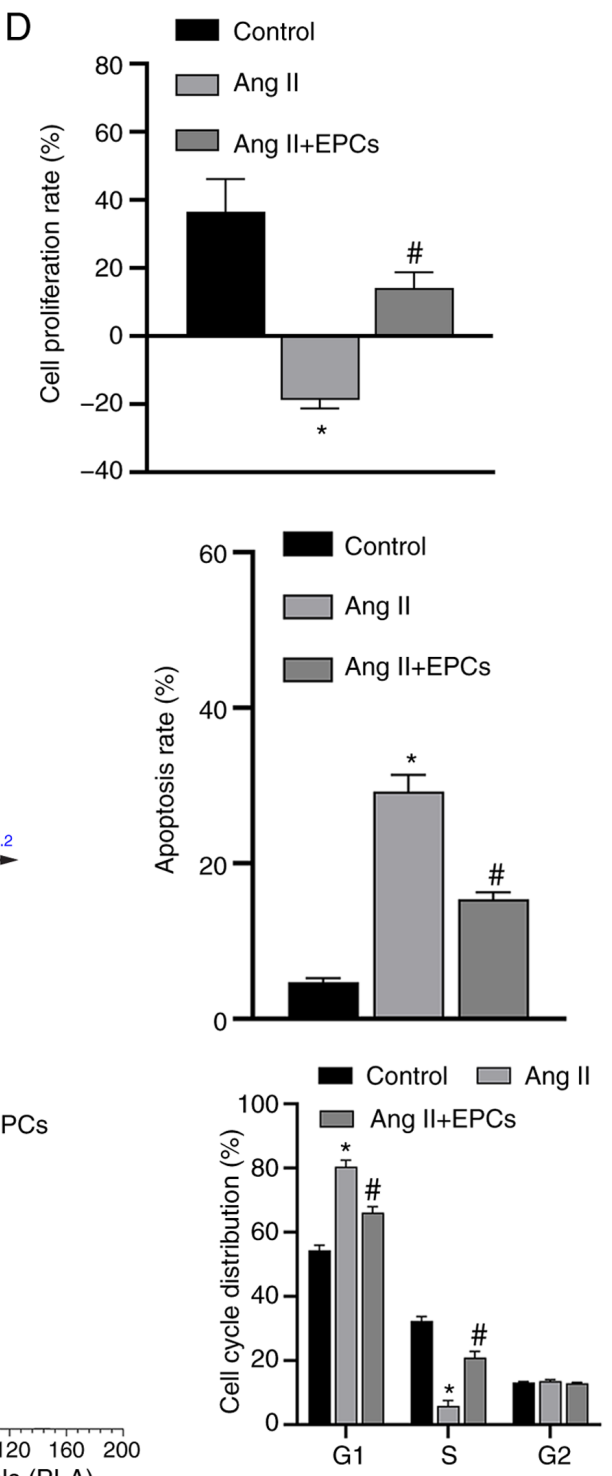

Figure 1. Recovery of PRKs by co-culture with EPCs following injury induced by Ang II. (A) Confirmation of the isolation of EPCs via Dil-Ac-LDL staining. Magnification, x100. (B) Confirmation of the isolation of PRKs using an immunofluorescence assay. Magnification, $\mathrm{x} 400$. (C) CCK-8 analysis of the effects of different proportions of co-cultured EPCs on the proliferation of PRKs. NS P>0.05 vs. PRKs:EPCs, 2:1 group; ${ }^{*}$ P<0.05 vs. PRKs:EPCs, 2:1 group. (D) CCK-8 analysis of the effects of co-cultured EPCs on the proliferation rate of Ang II-treated PRKs. FCM analysis of the effects of co-cultured EPCs on the (E) apoptosis and (F) cell cycle of Ang II-treated PRKs. ${ }^{*} \mathrm{P}<0.05$ vs. Control; ${ }^{*} \mathrm{P}<0.05$ vs. Ang II. Ang II, angiotensin II; EPCs, endothelial progenitor cells; PRKs, primary rat kidney cells; FCM, flow cytometry; CCK-8, Cell Counting Kit-8; Dil-Ac-LDL, Dil complex acetylated low-density lipoprotein; OD, optical density; $\alpha$-SMA, $\alpha$-smooth muscle actin; NS, not significant.

opposite results with regards to the secretion of these enzymes. The generation of ROS may therefore be involved in Ang II-induced cell damage, and can be ameliorated by co-culture with EPCs. 

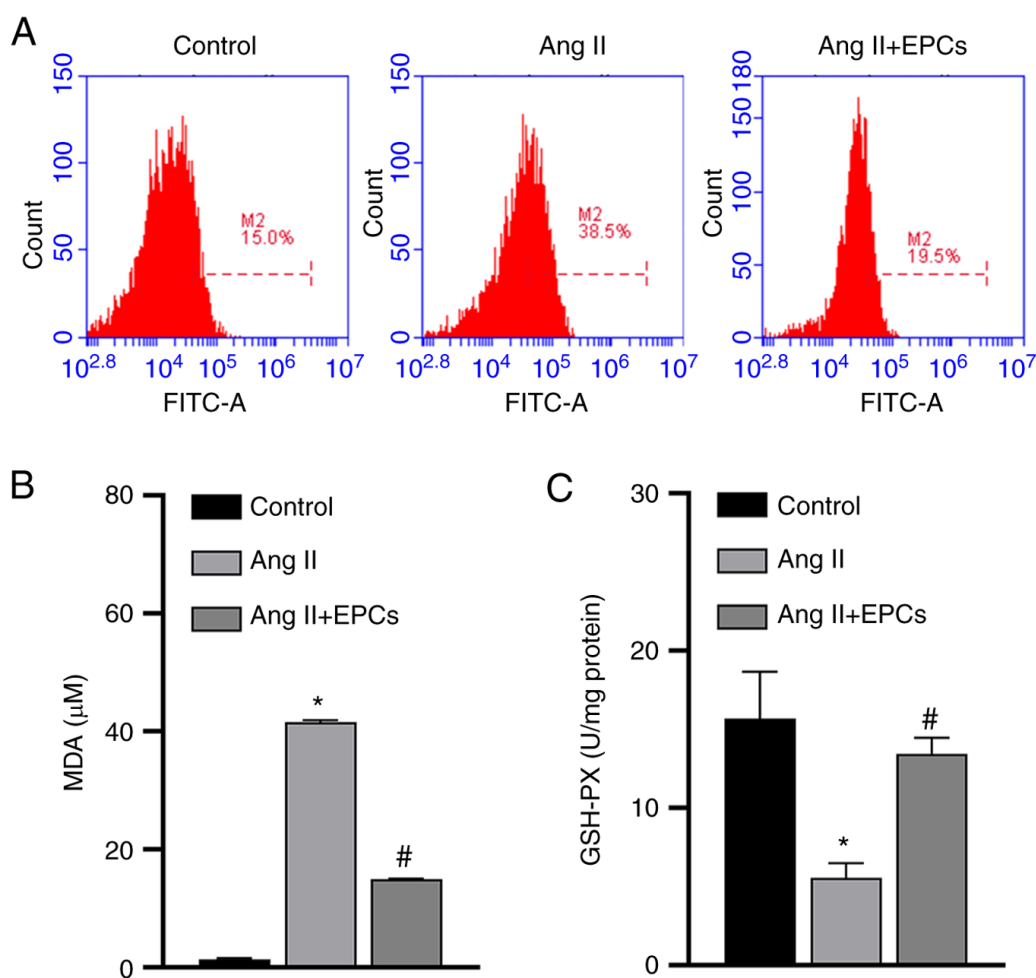

E

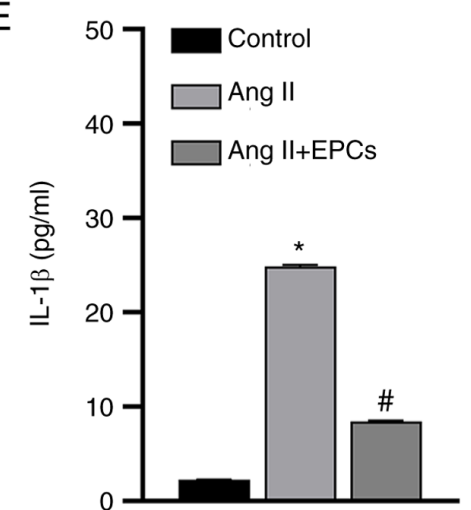

$\mathrm{F}$

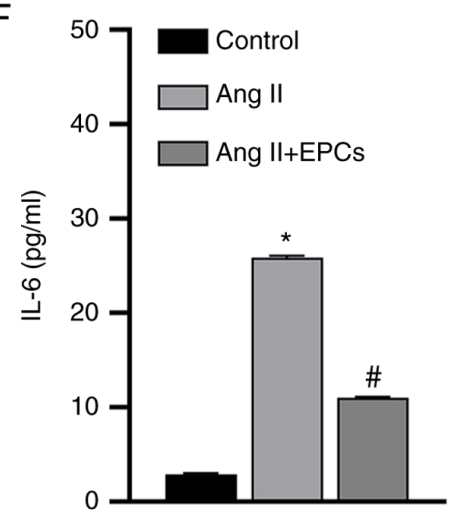

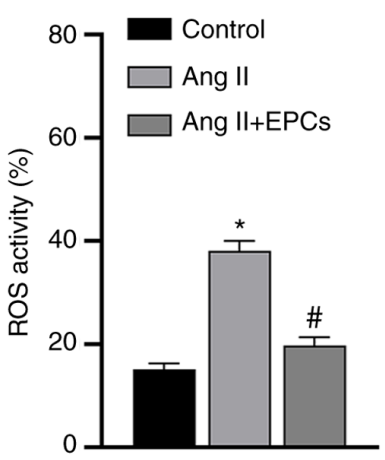
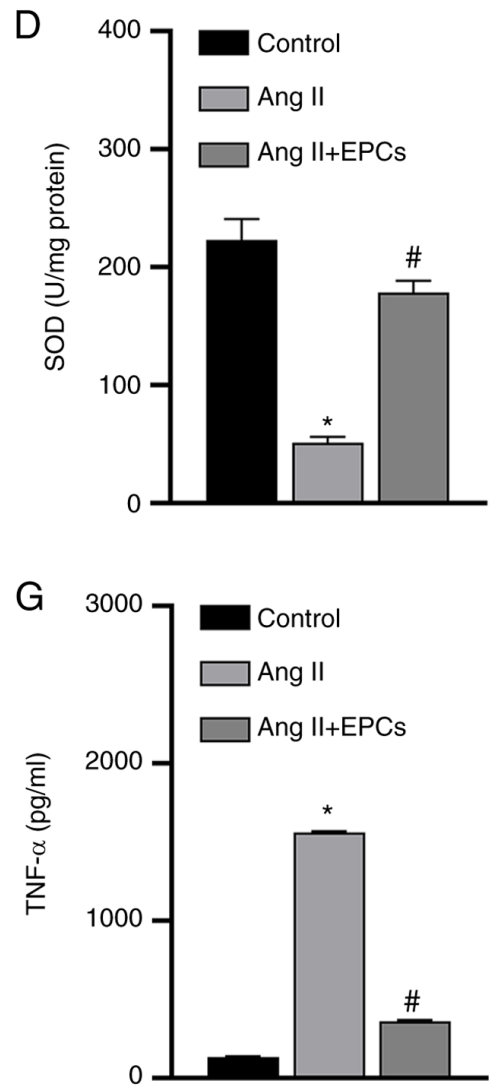

Figure 2. EPCs inhibit oxidative stress and secretion of inflammatory cytokines in a PRK kidney injury model induced by Ang II. (A) Flow cytometry analysis of the effect of EPC co-culture on the levels of ROS produced by Ang II-treated PRKs. ELISA results of the effect of EPCs on the levels of (B) MDA, (C) GSH-Px and (D) SOD in Ang II-treated PRKs. Effect of EPC co-culture on the levels of secreted (E) IL-1 $\beta$, (F) IL-6 and (G) TNF- $\alpha$ in Ang II-treated PRKs. " $\mathrm{P}<0.05$ vs. Control; ${ }^{*} \mathrm{P}<0.05$ vs. Ang II. Ang II, angiotensin II; ROS, reactive oxygen species; EPCs, endothelial progenitor cells; PRKs, primary rat kidney cells; MDA, malondialdehyde; GSH-Px, glutathione peroxidase; SOD, superoxide dismutase.

Ang II can also damage cells by promoting the secretion of inflammatory cytokines, while EPCs can prevent inflammation (32). Next, it was investigated whether Ang II promotes the secretion of inflammatory cytokines in PRKs, and whether EPCs could exert their protective role by inhibiting the secretion of inflammatory cytokines. The secretion of IL-1 $\beta$, IL- 6 and TNF- $\alpha$ was significantly upregulated in PRKs by the addition of Ang II. By contrast, in PRKs co-cultured with EPCs, the levels of IL- $1 \beta$, IL- 6 and TNF- $\alpha$ (Fig. 2E-G) were comparatively lower $(\mathrm{P}<0.05)$ than those in PRKs cultured alone. Therefore, the damage induced by Ang II in PRKs was also associated with pro-inflammatory cytokines, and co-culture with EPCs exerted protective effects by reducing the secretion of these cytokines.
Protective effect of EPCs is exerted via MVs. The mechanisms via which EPCs exert protective effects on Ang II-induced kidney cells were further analyzed by isolating MVs from EPCs (Fig. 3A). EPC-MVs were labeled with PKH26 and added to PRKs along with Ang II in a Transwell plate, as previously described. PKH26 fluorescence was detected in the cytoplasm of PRKs (Fig. 3B), indicating that EPC-MVs had fused with PRKs. Then, it was found that Ang II-induced decreased proliferation rate, promoted apoptosis and cycle arrest in the $\mathrm{G}_{1}$ phase, were inhibited by EPC-MVs (Fig. 3C-E). Similar to PRKs co-cultured with EPCs, PRKs grown in the presence of EPC-MVs and exposed to Ang II had reduced levels of ROS and MDA (Fig. 4A and B), increased GSH and SOD 
A

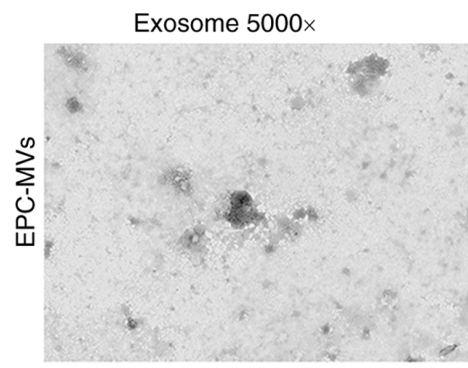

Exosome 15000x

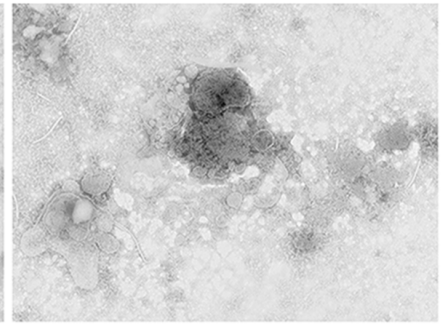

B

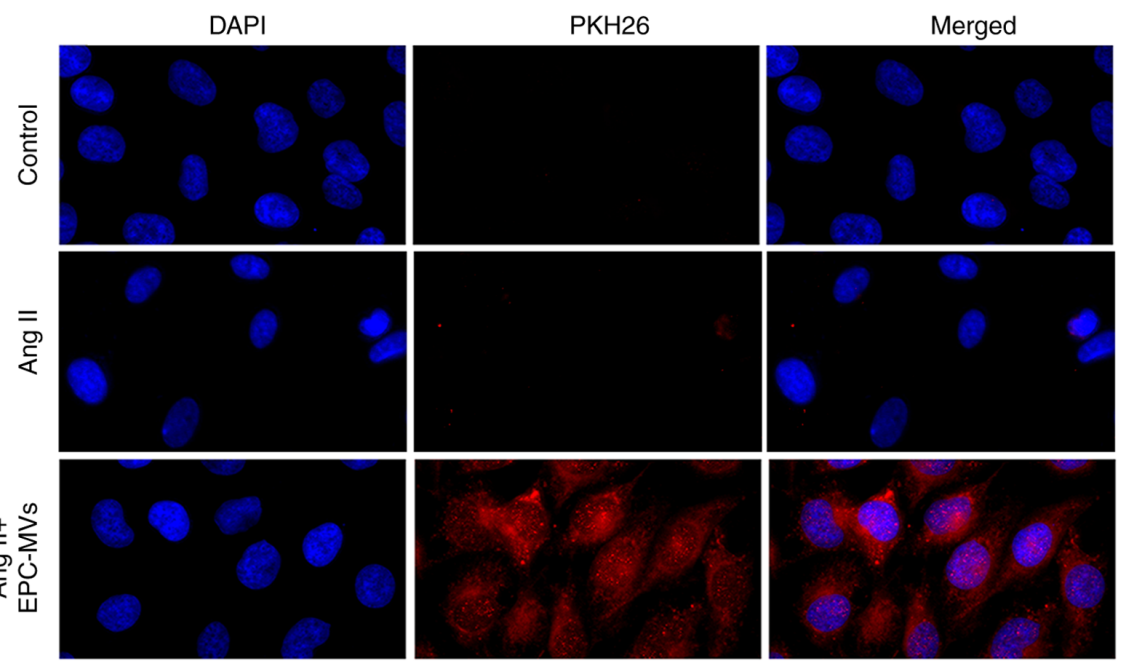

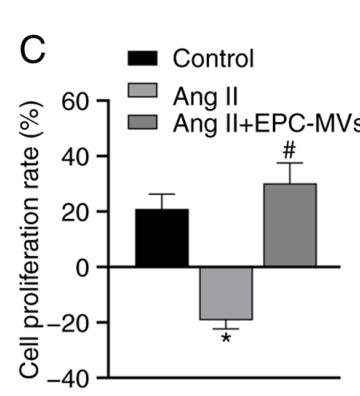

E

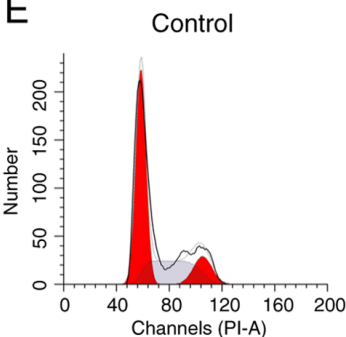

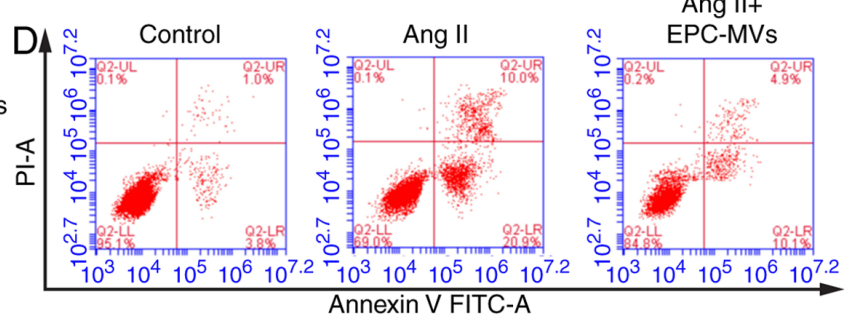

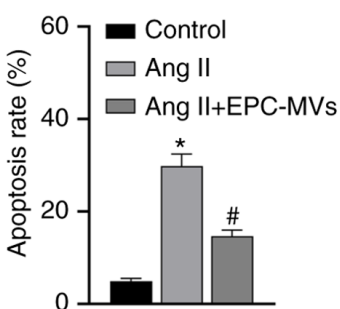

Control $\square$ Ang II

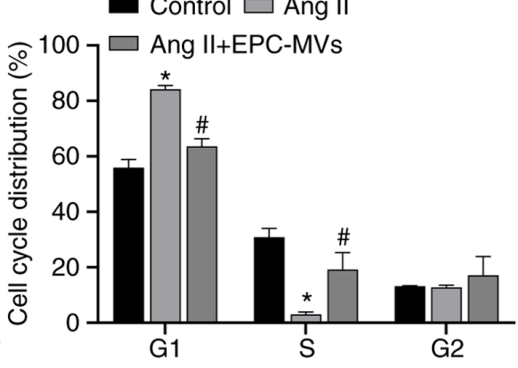

Figure 3. Recovery of PRKs by co-culture with EPC-MVs following injury induced by Ang II. (A) Successful isolation of EPC-MVs from EPCs as determined via transmission electron microscopy. Magnification, x5,000 and x15,000. (B) Fusion of PKH26- labeled EPC-MVs with PRKs, Magnification, x400. (C) CCK-8 analysis of the effects of different proportions of co-cultured EPC-MV on the proliferation of PRKs. Flow cytometry analysis of the effects of co-cultured EPC-MVs on the (D) apoptosis and (E) cell cycle of Ang II-treated PRKs. " $\mathrm{P}<0.05$ vs. Control; " $\mathrm{P}<0.05$ vs. Ang II. Ang II, angiotensin II; EPCs, endothelial progenitor cells; PRKs, primary rat kidney cells; MVs, microvesicles.

levels (Fig. 4C and D) and decreased secretion of the inflammatory cytokines IL-1 $\beta$, IL-6 and TNF- $\alpha$ (Fig. 4E-G), compared with the Ang II group.

\section{Discussion}

To the best of our knowledge, the present study demonstrated for the first time that EPCs can protect PRKs from Ang II-induced cell damage. This protective effect is, at least partly, mediated by MVs that are secreted by EPCs and fuse with PRKs during co-culture. By adding enriched MVs to PRKs exposed to Ang II, it was found that EPC-MVs alone could protect PRKs from Ang II-induced damage by inhibiting oxidative stress and inflammation.

MVs are defined as membrane nanodebris (0.05-1 $\mu \mathrm{m})(33)$, and are shed from the cell surface after activation, stress or 

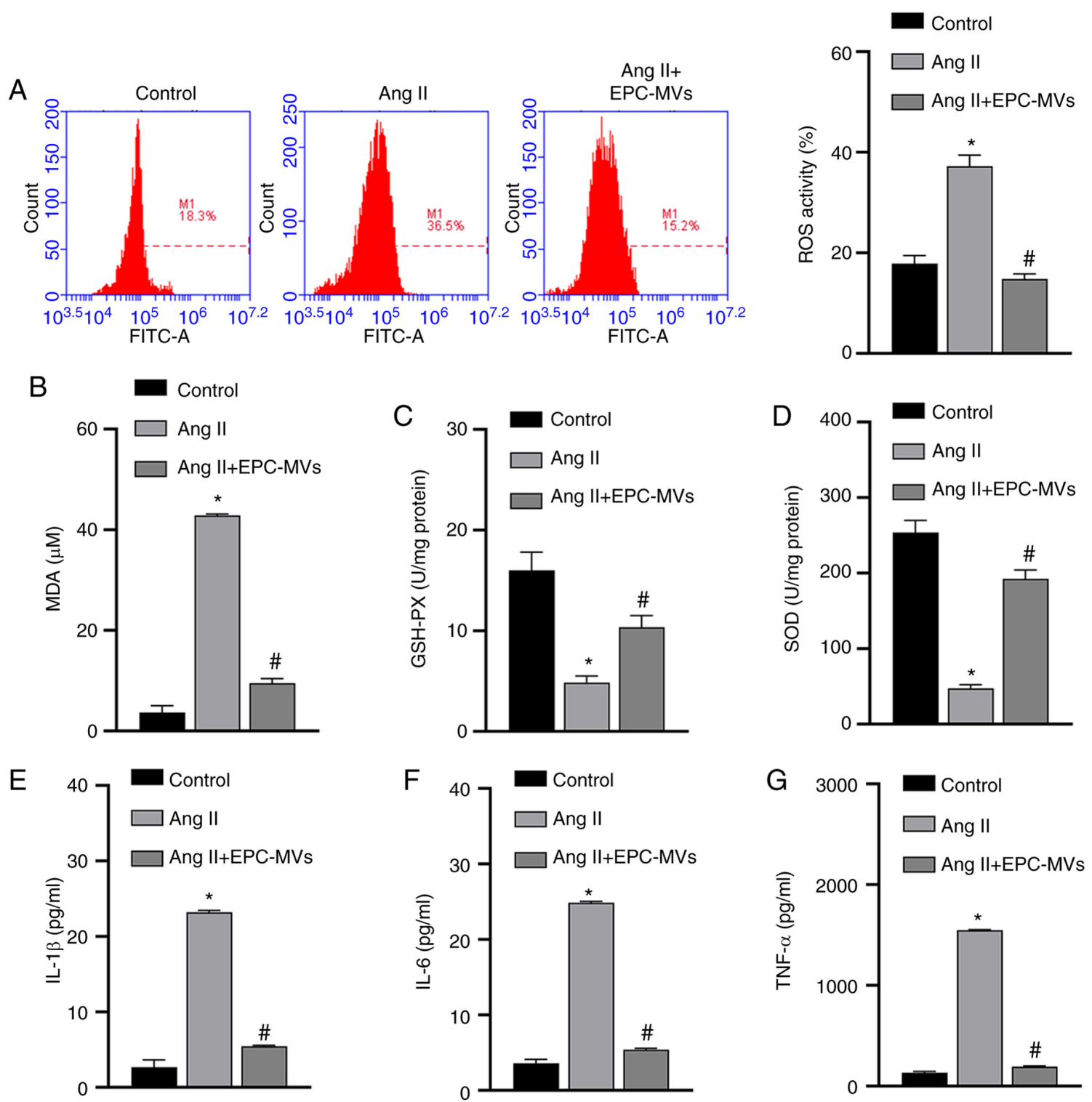

Figure 4. EPCs ameliorate the oxidative stress and inflammation induced by Ang II in PRKs via secreted MVs. (A) Flow cytometry analysis to determine the effect of EPC-MVs on ROS generation in Ang II-treated PRKs. ELISA results of the effect of EPC-MVs on the levels of (B) MDA, (C) GSH and (D) SOD in Ang II-treated PRKs. The effect of EPC-MVs on the levels of (E) IL-1 $\beta$, (F) IL-6 and (G) TNF- $\alpha$ secreted by Ang II-treated PRKs. "P<0.05 vs. Control; ${ }^{\text {"P }}<0.05$ vs. Ang II. Ang II, angiotensin II; ROS, reactive oxygen species; EPCs, endothelial progenitor cells; PRKs, primary rat kidney cells; MVs, microvesicles; MDA, malondialdehyde; GSH, glutathione; SOD, superoxide dismutase.

apoptosis. Furthermore, MVs can be derived from various cell types, such as platelets, endothelial cells, EPCs and white blood cells $(34,35)$. MVs express specific cell surface markers, which vary with the origin cell and their formation, and can exert anti-inflammatory, anticoagulant and angiogenic effects (36). MVs released from EPCs can carry the biological information of their parent cell, and thus exert a similar function on the target cells (33). For example, EPC-MVs protect cardiomyocytes from Ang II-induced hypertrophy and apoptosis (28), improve endothelial function and the ability to regulate angiogenesis (37), and alleviate endothelial dysfunction induced by oxidative stress (38). However, to the best of our knowledge, the effect of EPC-MVs on hypertensive nephropathy has not been previously reported. Therefore, the present study aimed to investigate the potential protective effects of EPCs and EPC-MVs in renal cell injury.
In the present study, a PRK injury model was established by inducing damage with Ang II. Consistent with previous reports $(26,39)$, Ang II reduced PRK proliferation, increased apoptosis and arrested the cell cycle at the $\mathrm{G}_{1}$ phase before entering the $\mathrm{S}$ phase. Based on these hypertension-related markers, it was concluded that the Ang II-induced PRK hypertension model was successfully established. Furthermore, it was determined that co-culture with EPCs protected PRKs from Ang II-induced damage. After co-incubation of EPCs with PRKs + Ang II, the levels of oxidative stress enzymes and inflammatory factors in the PRKs were decreased. Thus, the potential mechanisms via which EPCs exert their protective effects on PRKs may involve the regulation of oxidative stress and inflammation. To the best of our knowledge, the current study demonstrated for the first time that EPCs can ameliorate Ang II-induced oxidative stress response and inflammation in PRKs. 
EPCs effectively protect renal function in CKD (40) and serve a major role in maintaining vascular integrity and repairing endothelial injury (15), as well as reduce vascular leakage, improve organ function and increase survival rate in sepsis (41). Accumulating evidence suggests that EPCs may exert a protective role via the secretion of MVs $(37,42,43)$.

To test the protective effect of EPC-MVs in PRKs, EPC-MVs were isolated and labeled with PKH26, and then the labeled MVs were co-incubated with PRKs + Ang II. The EPC-MVs fused with PRKs and significantly inhibited the oxidative stress response and inflammation induced by Ang II, and these results are similar to those of Fu et al (44). The inhibitory effect of EPC-MVs on the levels of oxidative stress enzymes was greater than that of EPCs alone; this can be attributed to the MVs being present at a higher concentration in the PRKs treated with EPC-MVs than in those co-cultured with EPCs. These findings suggests that the protective effect of EPCs on PRKs against Ang II-induced cell damage may depend on secreted MVs.

A limitation of the present study is that the potential mechanisms via which the EPC-MVs regulate microRNAs/mRNAs/signal transduction axes were not fully examined. The role and mechanisms via which EPC-MVs exert protection against kidney damage should be evaluated in an animal model of hypertensive nephropathy, which is a promising direction for future research.

In conclusion, the present study demonstrated that EPCs can protect PRKs from Ang II-induced oxidative stress and increased inflammation via the secretion of MVs. This provides a novel direction for the treatment of hypertensive nephropathy, and establishes an in vitro model for studying kidney injury.

\section{Acknowledgements}

Not applicable.

\section{Funding}

This study was supported by the Finance Science and Technology Projects of Hainan Province (grant no. ZDYF2019193), National Natural Science Foundation of China (grant no. 8156020231) and CAMS Innovation Fund for Medical Sciences (grant no. 2019-I2M-5-023).

\section{Availability of data and materials}

The data used and/or analyzed during the current study are available from the corresponding author on reasonable request.

\section{Authors' contributions}

YS and ZB conceived the study, and SG designed the study and developed the methodology. YS, ZB, YZ, JC, MC, YZ and $\mathrm{XZ}$ performed the experiments and collected the data. $\mathrm{ZB}, \mathrm{HM}, \mathrm{BW}$ and YL analyzed and interpreted the data. YS and ZB wrote the original draft. All authors read and approved the final manuscript. YS, ZB, and SG confirmed the authenticity of all the raw data.

\section{Ethics approval and consent to participate}

All animal experiments were approved by the Animal Care and Use Committee of Hainan Medical University (Haikou, China; approval no. HYLL-2021-053), and were performed according to the National Institutes of Health guidelines.

\section{Patient consent for publication}

Not applicable.

\section{Competing interests}

The authors declare that they have no competing interests.

\section{References}

1. Strain WD and Paldánius PM: Diabetes, cardiovascular disease and the microcirculation. Cardiovasc Diabetol 17: 57, 2018.

2. Zhang L, Wang F, Wang L, Wang W, Liu B, Liu J, Chen M, He Q, Liao Y, Yu X, et al: Prevalence of chronic kidney disease in China: A cross-sectional survey. Lancet 379: 815-822, 2012.

3. Saran R, Robinson B, Abbott KC, Agodoa LYC, Bhave N, Bragg-Gresham J, Balkrishnan R, Dietrich X, Eckard A, Eggers PW, et al: US Renal Data System 2017 Annual Data Report: Epidemiology of kidney disease in the United States. Am J Kidney Dis 71 (Suppl 1): A7, 2018.

4. Hart PD and Bakris GL: Hypertensive nephropathy: Prevention and treatment recommendations. Expert Opin Pharmacother 11: 2675-2686, 2010

5. Xu W, Hu X, Qi X, Zhu R, Li C, Zhu Y, Yin S, Cheng L, Zhu R and Vitamin D: Vitamin D Ameliorates Angiotensin II-Induced Human Endothelial Progenitor Cell Injury via the PPAR- $\gamma / \mathrm{HO}-1$ Pathway. J Vasc Res 56: 17-27, 2019.

6. Feng X, Wang L and Li Y: Change of telomere length in angiotensin II-induced human glomerular mesangial cell senescence and the protective role of losartan. Mol Med Rep 4: 255-260, 2011.

7. Montezano AC, Nguyen Dinh Cat A, Rios FJ and Touyz RM: Angiotensin II and vascular injury. Curr Hypertens Rep 16: 431, 2014.

8. Dong T, Chen JW, Tian LL, Wang LH, Jiang RD, Zhang Z, Xu JB, Zhao XD, Zhu W, Wang GQ, et al: Role of the renin-angiotensin system, renal sympathetic nerve system, and oxidative stress in chronic foot shock-induced hypertension in rats. Int J Biol Sci 11: 652-663, 2015.

9. Zhang H, Zhang S, Jia L and Li H: MyD88 overexpression deteriorates Ang-II-induced ED via upregulating MPO and COX2 and downregulating eNOS in the corpus cavernosum of rats. J Cell Biochem: Nov 28, 2018 (Epub ahead of print). doi: 10.1002/ jcb.27987.

10. Zhang L, Du J, Hu Z, Han G, Delafontaine P, Garcia G and Mitch WE: IL- 6 and serum amyloid A synergy mediates angiotensin II-induced muscle wasting. J Am Soc Nephrol 20: 604-612, 2009.

11. Zhang LL,Huang S, MaXX,Zhang WY, Wang D, Jin SY,Zhang YP, Li Y and Li X: Angiotensin(1-7) attenuated Angiotensin II-induced hepatocyte EMT by inhibiting NOX-derived $\mathrm{H}_{2} \mathrm{O}_{2}$-activated NLRP3 inflammasome/IL-1 $\beta /$ Smad circuit. Free Radic Biol Med 97: 531-543, 2016

12. Liu YS, Yang Q, Li S, Luo L, Liu HY,Li XY and Gao ZN: Luteolin attenuates angiotensin II induced renal damage in apolipoprotein $\mathrm{E}$ deficient mice. Mol Med Rep 23: 1, 2021.

13. Mohammed-Ali Z, Cruz GL, Lu C, Carlisle RE, Werner KE, Ask K and Dickhout JG: Development of a model of chronic kidney disease in the C57BL/6 mouse with properties of progressive human CKD. BioMed Res Int 2015: 172302, 2015.

14. Souza AC, Tsuji T, Baranova IN, Bocharov AV, Wilkins KJ, Street JM, Alvarez-Prats A, Hu X, Eggerman T, Yuen PS, et al: TLR4 mutant mice are protected from renal fibrosis and chronic kidney disease progression. Physiol Rep 3: 3, 2015.

15. Ozkok A and Yildiz A: Endothelial Progenitor Cells and Kidney Diseases. Kidney Blood Press Res 43: 701-718, 2018.

16. Zhang G, Lin X, Shao Y, Su C, Tao J and Liu X: Berberine reduces endothelial injury and arterial stiffness in spontaneously hypertensive rats. Clin Exp Hypertens 42: 257-265, 2020. 
17. Hu H, Jiang C, Li R and Zhao J: Comparison of endothelial celland endothelial progenitor cell-derived exosomes in promoting vascular endothelial cell repair. Int J Clin Exp Pathol 12: 2793-2800, 2019.

18. Pompilio G, Capogrossi MC, Pesce M, Alamanni F, DiCampli C, Achilli F, Germani A and Biglioli P: Endothelial progenitor cells and cardiovascular homeostasis: Clinical implications. Int J Cardiol 131: 156-167, 2009.

19. Naito H, Iba T and Takakura N: Mechanisms of new blood-vessel formation and proliferative heterogeneity of endothelial cells. Int Immunol 32: 295-305, 2020.

20. Cantaluppi V, Gatti S, Medica D, Figliolini F, Bruno S, Deregibus MC, Sordi A, Biancone L, Tetta C and Camussi G: Microvesicles derived from endothelial progenitor cells protect the kidney from ischemia-reperfusion injury by microRNA-dependent reprogramming of resident renal cells. Kidney Int 82: 412-427, 2012.

21. Di Marco GS, Rustemeyer P, Brand M, Koch R, Kentrup D, Grabner A, Greve B, Wittkowski W, PavenstädtH,Hausberg M, et al: Circulating endothelial progenitor cells in kidney transplant patients. PLoS One 6: e24046, 2011.

22. Panagiotou N, Wayne Davies R, Selman C and Shiels PG: Microvesicles as vehicles for tissue regeneration: Changing of the guards. Curr Pathobiol Rep 4: 181-187, 2016.

23. Ranghino A, Cantaluppi V, Grange C, Vitillo L, Fop F Biancone L, Deregibus MC, Tetta C, Segoloni GP and Camussi G: Endothelial progenitor cell-derived microvesicles improve neovascularization in a murine model of hindlimb ischemia. Int J Immunopathol Pharmacol 25: 75-85, 2012.

24. Zhang M, Malik AB and Rehman J: Endothelial progenitor cells and vascular repair. Curr Opin Hematol 21: 224-228, 2014

25. Shipp AC and Patterson AP: The National Institutes of Health system for enhancing the science, safety, and ethics of recombinant DNA research. Comp Med 53: 159-164, 2003.

26. Tan YS and Lei YL: Isolation of tumor-infiltrating lymphocytes by ficoll-paque density gradient centrifugation. Methods Mol Biol 1960: 93-99, 2019.

27. Gyabaah K, Aboushwareb T, Guimaraes Souza N, Yamaleyeva L, Varner A, Wang HJ, Atala A and Yoo JJ: Controlled regulation of erythropoietin by primary cultured renal cells for renal failure induced anemia. J Urol 188: 2000-2006, 2012.

28. Elliget KA and Trump BF: Primary cultures of normal rat kidney proximal tubule epithelial cells for studies of renal cell injury. In Vitro Cell Dev Biol 27A: 739-748, 1991.

29. Nair AR, Ebenezer PJ, Saini Y and Francis J: Angiotensin IIinduced hypertensive renal inflammation is mediated through HMGB1-TLR4 signaling in rat tubulo-epithelial cells. Exp Cell Res 335: 238-247, 2015.

30. Giles EM, Godbout C, Chi W, Glick MA, Lin T, Li R, Schemitsch EH and Nauth A: Subtypes of endothelial progenitor cells affect healing of segmental bone defects differently. Int Orthop 41: 2337-2343, 2017.

31. Gu S, Zhang W, Chen J, Ma R, Xiao X, Ma X, Yao Z and Chen Y: EPC-derived microvesicles protect cardiomyocytes from Ang II-induced hypertrophy and apoptosis. PLoS One 9: e85396, 2014.
32. Xu X, Yang J, Li N, Wu R, Tian H, Song H and Wang H: Role of Endothelial progenitor cell transplantation in rats with sepsis. Transplant Proc 47: 2991-3001, 2015.

33. Meziani F, Tesse A and Andriantsitohaina R: Microparticles are vectors of paradoxical information in vascular cells including the endothelium: Role in health and diseases. Pharmacol Rep 60: 75-84, 2008.

34. Lee Y,El Andaloussi S and Wood MJ: Exosomes and microvesicles: Extracellular vesicles for genetic information transfer and gene therapy. Hum Mol Genet 21: R125-R134, 2012.

35. Martínez MC, Tesse A, Zobairi F and Andriantsitohaina R: Shed membrane microparticles from circulating and vascular cells in regulating vascular function. Am J Physiol Heart Circ Physiol 288: H1004-H1009, 2005.

36. McVey M, Tabuchi A and Kuebler WM: Microparticles and acute lung injury. Am J Physiol Lung Cell Mol Physiol 303: L364-L381, 2012.

37. Zeng W, Lei Q, Ma J and Ju R: Effects of hypoxic-ischemic pre-treatment on microvesicles derived from endothelial progenitor cells. Exp Ther Med 19: 2171-2178, 2020

38. Wang J, Chen S, Ma X, Cheng C, Xiao X, Chen J, Liu S, Zhao B and Chen Y: Effects of endothelial progenitor cell-derived microvesicles on hypoxia/reoxygenation-induced endothelial dysfunction and apoptosis. Oxid Med Cell Longev 2013: 572729 , 2013.

39. Wolak T, Kim H, Ren Y, Kim J, Vaziri ND and Nicholas SB: Osteopontin modulates angiotensin II-induced inflammation, oxidative stress, and fibrosis of the kidney. Kidney Int 76: 32-43, 2009.

40. Sung PH, Chen KH, Li YC, Chiang JY, Lee MS and Yip HK Sitagliptin and shock wave-supported peripheral blood derived endothelial progenitor cell therapy effectively preserves residual renal function in chronic kidney disease in rat-role of dipeptidyl peptidase 4 inhibition. Biomed Pharmacother 111: 1088-1102, 2019.

41. Zhou Y, Li P, Goodwin AJ, Cook JA, Halushka PV, Chang E and Fan H: Exosomes from endothelial progenitor cells improve the outcome of a murine model of sepsis. Mol Ther 26: 1375-1384, 2018.

42. Zeng W, Lei Q, Ma J, Gao S and Ju R: Endothelial progenitor cell-derived microvesicles promote angiogenesis in rat brain microvascular endothelial cells in vitro. Front Cell Neurosci 15: 638351, 2021

43. Cantaluppi V, Biancone L, Figliolini F, Beltramo S, Medica D, Deregibus MC, Galimi F, Romagnoli R, Salizzoni M, Tetta C, et al: Microvesicles derived from endothelial progenitor cells enhance neoangiogenesis of human pancreatic islets. Cell Transplant 21: 1305-1320, 2012.

44. Fu C, Chen B, Jin X, Liu X, Wang F, Guo R, Chen Z, Zheng H, Wang L and Zhang Y: Puerarin protects endothelial progenitor cells from damage of angiotensin II via activation of ERK1/2 Nrf2 signaling pathway. Mol Med Rep 17: 3877-3883, 2018

(i) $\ominus$ This work is licensed under a Creative Commons Attribution-NonCommercial-NoDerivatives 4.0 International (CC BY-NC-ND 4.0) License. 